\title{
Working Memory and Flexibility in creative thinking
}

\author{
Jarosław Orzechowski
}

\begin{abstract}
The current study is focused on the relationship between working memory and flexibility of thinking (as one of Guilfordian components of divergent thinking). The empirical research consisted of two experiments that used an adaptation of the Figural Similarities Test (based on Guilford). Participants' task was to search for as many classes composed of three figures (selected from a set of six figures) as possible. It was assumed that manipulation of the number of defining elements (visual complexity) would influence the load of the control system, whereas manipulation of the number of relations (relational complexity) the load of working memory. Results show that visual complexity of the material loads the control system with the costs of inhibiting insignificant features of the stimulus. Whereas the relational complexity loads the storage mechanism with the necessity to maintain a greater number of relations. Moreover, this is compounded by the necessity to reduce complexity of the task, if the number of relations to consider resulting from the complexity of the stimulus (Experiment 1) or the number of relations that must be simultaneously taken into account (Experiment 2) exceeds cognitive abilities of the individual. Thus, the obtained results indicate complex relationships between WM and flexibility in divergent thinking.
\end{abstract}

Keywords — creativity, divergent thinking, flexibility, working memory

\section{INTRODUCTION}

$\mathrm{W}$ ORKING memory (WM) is regarded as the engine of the mind. So it is not surprising that research on the impact of working memory capacity (WMC) on intellectual functioning has been conducted for many years. Whereas the relationship between WMC and intelligence has been repeatedly replicated, studies on the role of WM in creative thinking are not conclusive.

For example, Ash and Wiley [1] tested the relationship between WM performance and the efficiency of solving insight tasks. It is assumed that in insight tasks a restructuring of the problem must occur. This restructuring is - usually - preceded by an impasse caused by searching of an

Jarosław Orzechowski is with the Institute of Psychology, Jagiellonian University, Poland (corresponding author's phone: +48501041051; e-mail: j.orzechowski@uj.edu.pl).

Research presented in the paper was financially supported by a grant from the State Committee for Scientific Research (no. PB 5 H01F 006 21) to Jarosław Orzechowski. Preparation of the paper was financially supported by a grant from the National Science Centre (no. DEC-2013/09/B/HS6/02649) to Jarosław Orzechowski. inappropriate problem space resulting directly from the original formulation of the problem. Experimentally, the researchers isolated two phases: (1) initial searching of the problem space, and (2) the restructuring of the task. The obtained results indicated significant participation of WMC in solving insight problems, insofar as both phases were present in the task. However, when only the phase 2 (the restructuring of the task) was isolated, by providing the participants with information enabling them to eliminate inappropriate "clues" in the phase 1, this effect disappeared. Also DeYoung, Flanders, and Peterson [2] looked for predictors of efficiency of solving insight problems. In their research, they took into account verbal intelligence, working memory, divergent thinking and the ability to break patterns. The results indicated that all of these factors influence solving of insight problems, but after isolating the variance unique to insight problems (by juxtaposing them with non-insight problems) it was found that only divergent thinking and the ability to break patterns remained significant predictors. In other studies [3], creativity was predicted by updating and inhibition (as executive processes of WM). Updating was also observed to explain a significant portion of shared variance between intelligence and creativity.

Researchers emphasize, however, that creative thinking is not a homogeneous construct. For example, Lin and Lien [4] looked for relationships between WM and divergent tasks and insight tasks, separately. They obtained a positive correlation between WMC and solving insight problems, but found no correlation between WMC and divergent tasks. According to the authors, this finding suggested that open-ended and closed-ended creative problem solving involved different processes and helped to clarify some past inconsistencies when considering the relationship between WM and creativity. Many studies show that relationships between WM and divergent thinking is not just positive, but quite strong. For example, interesting results concerning relationships between performing tasks that require divergent thinking and an n-back task, with the use of the fMRI neuroimaging technique, were obtained by Takeuchi and his team [5]. Convergence of activation in various brain areas in a group of more creative persons (distinguished on the basis of a divergent task) and more efficient in a memory task was interpreted as ineffective allocation of attention in creative persons. Diffusion of attention would be a mechanism that 
favours including information remotely associated with the problem into creative tasks, and that would be conducive to generation of new solutions, which is not a new result [7]. [8].

Inconsistency in the research results persuaded the author to undertake a systematic search for a cognitive basis of the efficiency of divergent thinking, in reference to WMC. Oberauer [8] argues that creating new relations between elements representing a task in order to integrate them in a new structure (e.g. a solution to a divergent problem) involves executive functions, but the complexity of this new structure is limited by working memory span. This limitation applies to the number of items that can be simultaneously placed in the system responsible for coordination. A fundamental question arises: is the number of items (related to WMC) or the number of relations that can occur between these items (related to the efficiency of WM control) a significant predictor of divergent thinking? In order to answer this question, a divergent task was constructed allowing to manipulate selectively either the number of elements defining the task (with a constant number of relations), or the number of relations between them (with constant number of defining elements). This study was focused on the flexibility of thinking as one of Guilfordian components of divergent thinking [9].

\section{METHOD}

The study used an original adaptation of the Figural Similarities Test [9] that consisted in searching for as many classes composed of three figures (selected from a set of six figures) as possible. It was assumed that a class is created by abstracting one or more relations linking the chosen three elements. Therefore, the task requires relational reasoning searching for relations between three elements, when the material is so selected that in each trial it is possible to generate many such triples. Because each (or most) of them will be based on different relations between the chosen elements, Guilford assumed that this task required mainly flexibility of thinking.

A computerized version of the task, i.e. the Figural Similarities Computerized Task (FSCT), was developed in two variants. In the first variant, the number of elements defining particular figures used in the task was manipulated, whereas in the second variant - the number of relations on the basis of which it was possible to create a three-element class was manipulated. Regardless of the variant, the participants received a set of six figures. Their task was to find as many classes consisting of three chosen figures as possible.

In the FSCT-A task, involving manipulation of the number of defining elements, appropriate matrices were developed in which the number of elements was 3, 6, or 9. However, to create three-elements classes out of the presented matrices it was always sufficient to take into account only one feature, and consequently only one relation was the basis for creating a class. In other words, the number of elements - increasing as a result of manipulation - was always accompanied by only one classification relation (see Fig. 1).
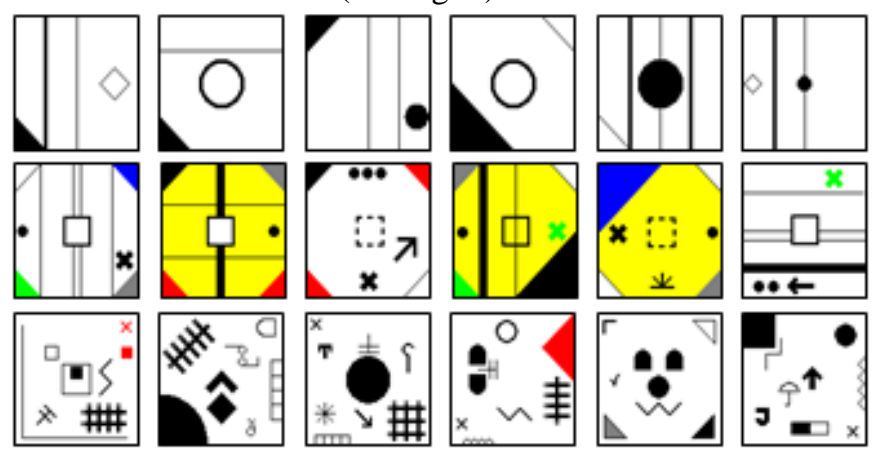

Fig. 1. Examples of FSCT-A Task involving manipulation of the number of defining elements: 3 (first row), 6 (second row), or 9 (third row).

In order to capture the impact of control processes on performing the FSCT task, in the middle of trials a secondary task was introduced. It was a simple motor task carried out simultaneously with the classification task. It consisted in keeping a little ball on the top of a "hill" on a computer screen. The difficulty was that the ball always rolled down to the right or to the left side of the "hill". It was assumed that the level of the secondary task performance is a measure of the efficiency of control exercised by the central executive system of WM. The measurement of deviation of the ball from its optimal position was made continuously in an attempt to capture the dynamics of changes in the level of control required by the priority task.

The task had 6 experimental conditions, resulting from the manipulation of the number of elements making up a figure $(3,6$, or 9) and two levels of the factor "the number of concurrent tasks" (one vs two tasks). In total, each participant carried out 18 tasks ( 6 conditions x 3 tasks) and two training tasks. After each answer, the participants entered justification of their choice in order to eliminate random arrangements.

The FSCT-B task had exactly the same experimental design, but the main manipulation concerned the number of relations forming a three-element class. At the same time, the number of elements defining a figure was 6 in the entire task. The tasks were so designed that the number of relations to be taken into account was 2, 3, or 4 (see Fig. 2). In the condition of two relations, one cannot build a correct class if one considers only one element of a figure (i.e. one relation only); in the condition of three elements - if one tries to use only one or two relations, etc. The possibility of creating classes based on more elements than specified in a given condition was also excluded. In the middle of trials the paradigm of concurrent tasks was again employed, similarly as in the variant A. Manipulation of the task variant (A or B) was carried out between groups. 


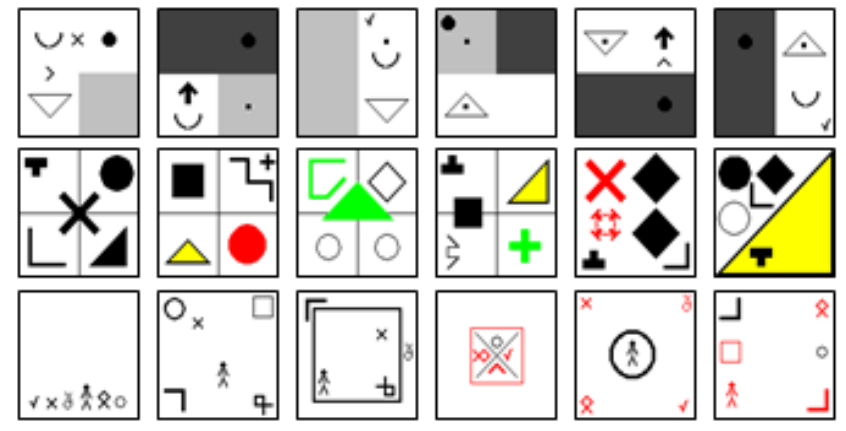

Fig. 2. Examples of FSCT-B Task involving manipulation of the number of relations forming a three-element class: 2 (first row), 3 (second row), or 4 (third row).

It was assumed that both manipulations - of the number of elements defining a figure and of the number of relations defining a class - can have an impact on the level of the task performance related to increasing WM load. However, each of them affects the involvement of memory and control subsystems of WM to a different degree. It was assumed that manipulation of the number of defining elements would, first of all, influence the load of the control system, whereas manipulation of the number of relations - the load of the memory system. In both cases, the secondary task would be an indicator of the efficiency of the control mechanism.

The first experiment, using the FSCT-A task, involved 50 participants (41 women and 9 men; age: 20.6 \pm 1.36 years). The second experiments, using the variant FSCT-B, involved 40 participants ( 31 women and 8 men; age: $20.65 \pm 1.7$ years).

\section{RESUlTS}

In both variants of the task, there was a significant impact of manipulation of the key factor differentiating the versions. In the FSCT-A task, the number of elements defining a figure influenced both the reaction time $(\mathrm{F}(2,82)=37.98, \mathrm{p}<0.0001$, see Fig. 3) and the number of correct solutions ( $F(2,82$ )=11.39, p<0.0001, see Fig. 4).

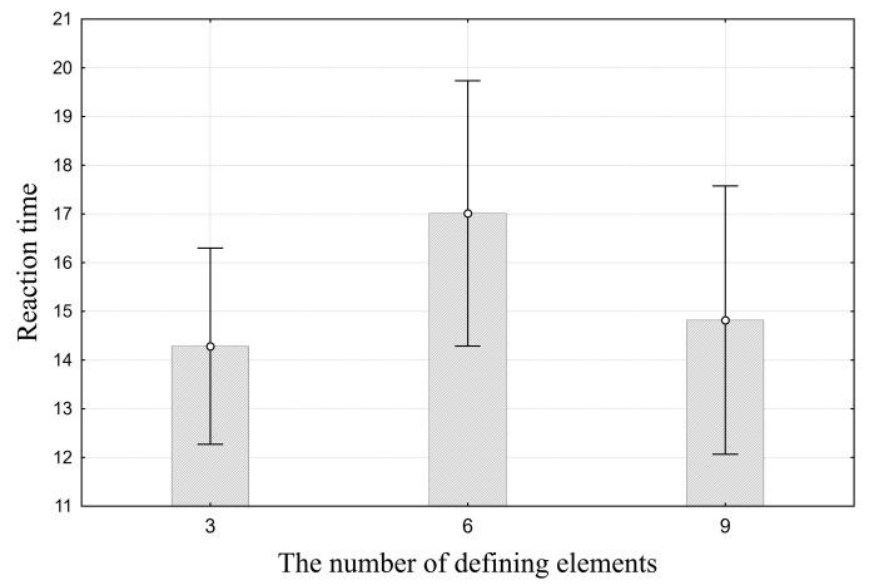

Fig. 3. Reaction time in the FSCT-A as a function of number of elements defining a figure.

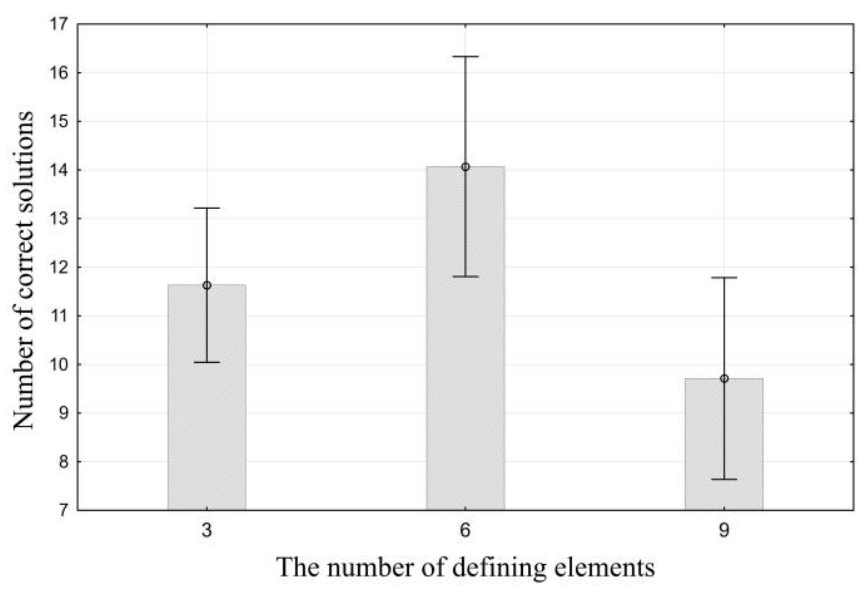

Fig. 4. Number of correct solutions in the FSCT-A as a function of number of elements defining a figure.

In both cases, the relation is curvilinear. Initially, the growing number of defining elements increases the reaction time and the number of discovered classes (with average WM load), and then their reduction (with maximum WM load).

In the FSCT-B task, there was a significant effect of the impact of the factor "the number of relations", but only on the correctness of solutions $(F(2,76)=4.49, p=0.015$, see Fig. 5). The increase of the number of relations that were to be taken into account in a given condition of the task initially causes an increase of the number of discovered classes, but subsequently - its decline.

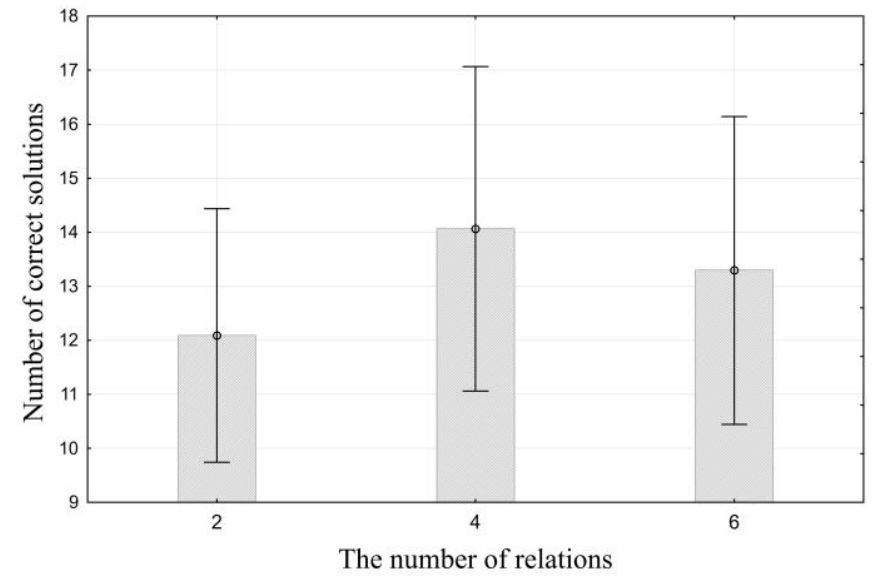

Fig. 5. Number of correct solutions in the FSCT-B as a function of number of relations between elements.

In both variants of the FSCT task a significant effect of the double task was also found. The effect consisted in decreasing the number of discovered classes in the condition of simultaneous tasks (FSCT-A: $\mathrm{F}(1,41)=21.75$, $\mathrm{p}<0.0001$; FSCT-B: $F(1,38)=10.21, \mathrm{p}=0.003)$. However, only in the variant $A$, the interaction of the factors "the number of elements defining a figure" and "the number of concurrent tasks" proved to be significant $(\mathrm{F}(2,82)=7.59, \mathrm{p}<0.001$, see Fig. 6). An analogous interaction of the factors the "number of relations defining a class" and "the number of concurrent tasks" in the variant B proved to be insignificant. 


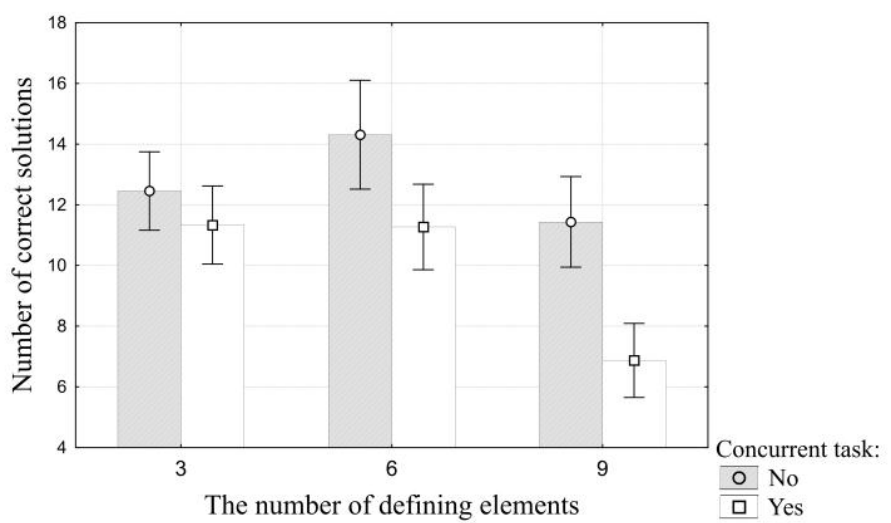

Fig. 6. Reaction time in the FSCT-A as a function of number of elements defining a figure and the number of concurrent tasks.

\section{DISCUSSION}

The obtained results indicate a relationship between the number of elements defining figures used in the FSCT-A task as well as the number of relations between the figures in the FSCT-B task and the results of figural flexibility obtained in the divergent task. However, in both cases the relations were curvilinear. The question is: why? It seems that the task was difficult even in its simplest variant, so the analysis of only three elements of a figure or two relations between them (Level I) led to WM overload. Further manipulation of both factors forced the participants to reduce the complexity of the classification task in order to make it doable. In the case of six defining elements or three relations (Level II), reduction of complexity led to a slight improvement of performance (more discovered classes), although in one of the variants (i.e. FSCT-A) it was at the cost of extending the reaction time. However, it seems that the complexity not always can be effectively reduced (Level III - nine elements or four relations). Such reduction - if we assume that it occurred at all - appears to be more costly for WM control processes in the condition of reduction of the number of defining elements, but not necessarily the number of relations. To be more precise, in both variants of the task the process of divergent production requires control (in both cases there was a difference in the correctness of classifications as a result of introduction of the secondary task), but only in the condition of the increasing number of elements, the cost of reduction of complexity is related to CE load.

One can cautiously assume that the complexity (here: the visual complexity) of the material loads the control system with the costs of inhibiting insignificant features of the stimulus. Whereas the relational complexity loads the storage mechanism with the necessity to maintain a greater number of relations. Moreover, this is compounded by the necessity to reduce complexity of the task, if the number of relations to consider resulting from the complexity of the stimulus (the FSCT-A task) or the number of relations that must be simultaneously taken into account (the FSCT-B task) exceeds cognitive abilities of the individual.

\section{V.CONCLUSION}

The obtained results indicate complex relationships between WM and flexibility in divergent thinking.

\section{REFERENCES}

[1] I. K. ASh AND J. Wiley, "The NATURE OF RESTRUCTURING IN INSIGHT: AN INDIVIDUAL - DIFFERENCES APPROACH," PSYCHONOMIC BULLETIN AND REVIEW, 13, PP. 66-73, 2006. https://doi.org/10.3758/BF03193814

[2] C. G. DeYoung, J. L. Flanders, and J. B. Peterson, "Cognitive abilities involved in insight problem solving: An individual differences model," Creativity Research Journal, 20, pp. 278-290, 2008. https://doi.org/10.1080/10400410802278719

[3] M. Benedek, E. Jauk, M. Sommer, M. Arendasy, and A. C. Neubauer, "Intelligence, creativity, and cognitive control: The common and differential involvement of executive functions in intelligence and creativity," Intelligence, 46, pp. 73-83, 2014. https://doi.org/10.1016/j.intell.2014.05.007

[4] W. L. Lin and Y. W. Lien, "The different role of working memory in openended versus closed-ended creative problem solving: a dual-process theory account," Creativity Research Journal, 25, pp. 85-96, 2013. https://doi.org/10.1080/10400419.2013.752249

[5] H. Takeuchi, Y. Taki, H. Hashizume, Y. Sassa, T. Nagase, R. Nouchi, and R. Kawashima, "Failing to deactivate: The association between brain activity during a working memory task and creativity," NeuroImage, 55, pp. 681-687, 2011. https://doi.org/10.1016/j.neuroimage.2010.11.052

[6] D. Rawlings (1985). "Psychotism, creativity, and dichotic shadowing," Personality and Individual Differences, 6, pp. 737-742, 1985. https://doi.org/10.1016/0191-8869(85)90084-4

[7] A. Stavridou and A. Furnham (1996), "The relationship between psychoticism, trait-creativity and the attentional mechanism of cognitive inhibition," Personality and Individual Differences, 21, pp. 143-153, 1996.

https://doi.org/10.1016/0191-8869(96)00030-X

[8] K. Oberauer, H. M. Süß, O. Wilhelm, and N. Sander, "Individual differences in working memory capacity and reasoning ability", In Variation in Working Memory, A. R. A. Conway, C. Jarrold, M. J. Kane, A. Miyake, and J. N. Towse, Eds, Oxford: Oxford University Press, 2007, pp. $49-75$.

[9] J. P. Guilford, The Nature of Human Intelligence. New York: McGrawHill, 1967. 